Service social

\title{
Du contrat pédagogique à l'évaluation dans un processus de supervision de groupe
}

\section{Christophe Dalibert}

Volume 46, numéro 2-3, 1997

Groupes - Symposium 1997

URI : https://id.erudit.org/iderudit/706765ar

DOI : https://doi.org/10.7202/706765ar

Aller au sommaire du numéro

Éditeur(s)

École de service social de l'Université Laval

ISSN

1708-1734 (numérique)

Découvrir la revue

Citer cet article

Dalibert, C. (1997). Du contrat pédagogique à l'évaluation dans un processus de supervision de groupe. Service social, 46(2-3), 167-184.

https://doi.org/10.7202/706765ar d'utilisation que vous pouvez consulter en ligne.

https://apropos.erudit.org/fr/usagers/politique-dutilisation/ 


\section{Du contrat pédagogique à l'évaluation dans un processus de supervision de groupe}

Christophe DALIBERT

L'intervention présentée dans ce texte se situe auprès de cinq professionnelles (assistantes de service social et conseillères en économie sociale et familiale), travaillant dans le dispositif du RMI (Revenu minimum d'insertion) dans une circonscription rurale de l'ouest de la France. Le Revenu minimum d'insertion est une allocation versée à toute personne sans ressources âgée de plus de 26 ans. Le RMI entre dans le champ de l'aide sociale obligatoire, le RMI est un droit. Depuis 1989, une tâche nouvelle s'est ajoutée au travail de ces professionnels : la réinsertion professionnelle. On peut lire en effet dans le texte de loi du 1er décembre 1988 et dans la circulaire du 9 mars 1989 : «Pour la plus grande part des bénéficiaires, la démarche d'insertion devra se fixer comme objectif à plus ou moins long terme l'insertion professionnelle, c'est-à-dire l'accession à un emploi [...] Au moment du dépôt de sa demande de RMI, l'intéressé doit souscrire l'engagement de participer aux activités ou actions d'insertion dont il sera convenu avec lui... ". Le rôle du professionnel est d'aider à la maturation du projet de réinsertion, voire de vérifier les possibilités de sa réalisation.

Ces travailleuses sociales sont salariées de trois institutions différentes : le département, la MSA (Mutualité sociale agricole) et un CCAS (Centre communal d'action sociale). Elles se réunissent régulièrement afin de mettre en place des réunions d'information collective à l'attention des usagers. 
Les usagers concernés par le dispositif RMI sont des personnes généralement en grandes difficultés tant financières que relationnelles. Ils sont pour la plupart à la recherche d'un premier emploi, en quête d'une insertion sociale, chômeurs de longue durée sans ressources ou presque.

Devant la difficulté grandissante de répondre de façon appropriée aux demandes des usagers, les professionnelles sont à la recherche de nouvelles méthodes de travail. Le besoin qu'elles ont de se regrouper trouve son origine dans l'isolement qu'elles ressentent dans leur travail au contact d'usagers qui font face à des situations de plus en plus dramatiques. Devant ces personnes en grande difficulté, l'intervention sociale se révèle délicate et difficile. Il en résulte un sentiment de solitude des travailleuses sociales, l'aide technique et le soutien de leurs encadrements respectifs ne correspondant pas à leurs attentes.

À la suite d'une journée d'information sur le travail social de groupe à laquelle ont participé certaines des professionnelles concernées, l'une d'entre elles me contacte pour l'aider dans la mise en place de groupes pour les usagers. Parallèlement à cette demande, les autres professionnelles souhaitent pouvoir bénéficier d'une formation au travail social de groupe.

\section{L'ACTION}

Mon intervention auprès du groupe de travailleuses sociales s'est déroulée sur six mois, à raison d'une rencontre de trois heures par mois. La demande initiale des professionnelles était de bénéficier d'une supervision pour la mise en place de groupes d'usagers.

\section{Les trois premières rencontres}

Devant la diversité des représentations que suscitait l'appellation " travail social de groupe ", il nous a fallu dans un premier temps faire un important travail de clarification des attentes de chacune, en faisant apparaître qu'il y avait entre elles des différences tant dans leurs attentes que dans la réalité quotidienne de leur travail. Étant d'institutions différentes, les populations auprès desquelles elles intervenaient (même si elles relevaient toutes du dispositif du RMI) étaient relativement différentes : certains usagers étaient issus du 
monde agricole, d'autres de la cité voisine, d'autres encore parachutés dans la région au bénéfice d'un logement bon marché qui s'était libéré.

Ce travail de clarification a permis de faire émerger les raisons qui amenaient ces professionnelles d'institutions différentes à se regrouper autour de leur travail et à rechercher de nouvelles approches méthodologiques.

Le sentiment de solitude dans l'accompagnement des usagers, l'impossibilité de répondre à des demandes qui ne relèvent par toujours de la compétence du travailleur social en exercice, l'impression de ne pas être entendue et d'être incomprise par une hiérarchie perçue comme loin des problèmes des usagers sont autant de raisons qui ont poussé ces professionnelles à se réunir.

Les motifs de leur regroupement sont également liés à la préparation et à l'organisation des réunions collectives d'information qui rassemblent une douzaine d'usagers afin de les aviser de leurs droits et devoirs dans le dispositif du RMI. La demande de supervision, dans l'esprit des travailleuses sociales, avait pour raison essentielle d'asseoir une pratique assez proche des réunions déjà existantes.

Les trois premières séances de travail ont rudement éprouvé les professionnelles. II s'est agi lors de ces rencontres de clarifier beaucoup de points obscurs. Sans un besoin important d'exprimer un fort malaise et sans une motivation remarquable des professionnelles, notre collaboration aurait pu s'achever prématurément.

Ainsi, il a fallu entendre et décrypter le malaise de ces travailleuses sociales. Pour ce faire, chacune d'entre elles s'est exprimée sur un cas qui lui posait problème. Après cet exercice, une des participantes a dit: " Jusque-là on parlait du RMI, maintenant on parle des RMlstes. " Les caractéristiques propres à tous les groupes ont fonctionné : l'écoute des pairs, la reconnaissance mutuelle, l'abandon du sentiment de solitude sur les situations...

Il a également fallu donner une information sur le travail social de groupe et faire bouger certaines représentations concernant la méthodologie et la finalité de cette méthode. Abandonner, par exemple, l'idée d'interchangeabilité telle qu'elle est pratiquée lors des réunions collectives d'information. Si le travail social collectif s'intéresse 
au résultat du groupe, en travail social de groupe le travailleur social est toujours attentif aux individus et à leur développement, c'est-àdire qu'il se préoccupe de l'épanouissement de chaque individu dans le groupe.

II a fallu préciser que le travail social de groupe ne s'appliquait pas à tout regroupement d'usagers, qu'avant de mettre en place un groupe il fallait mesurer les effets qu'aurait un tel travail. Que le professionnel se fasse plaisir, pourquoi pas, mais avant tout son souci premier doit être l'usager.

La rédaction du contrat, justifiant mon intervention auprès des professionnelles, était une chose à laquelle je tenais particulièrement. Toute cette phase de préparation et de clarification a contribué à ce que le contrat soit le plus pertinent possible. Ce contrat avait pour valeur essentielle de préciser aux travailleuses sociales le pourquoi, le comment et les limites de notre collaboration. II avait aussi valeur d'information de l'existence officielle du groupe et de son objet auprès des institutions des professionnelles présentes dans le groupe.

Avant de passer à la phase suivante, précisons que lors de ces premières rencontres, en plus de la méthodologie propre à la constitution des groupes et en plus de l'approche spécifique à la supervision, nous avons utilisé les techniques transitives de communication élaborées par William Schwartz et décrites par L. Shulman (1976).

\section{Les rencontres pour travailler aux projets}

Une des conditions établies avec les travailleuses sociales dès la première rencontre concemait la nécessité d'adresser un écrit concernant leur projet entre les réunions de travail. De ce fait, chacune a pu commencer à réfléchir à un projet plus personnel qu'elle pourrait mettre en place.

Lors des séances de travail, l'objet de notre réflexion s'est essentiellement centré sur les projets de chacune. Ces projets ont alors évolué très rapidement avec le développement de l'aide des professionnelles entre elles, ainsi qu'avec l'intérêt manifeste de chacune pour les projets des autres. Á cette étape du processus, j'ai utilisé en tant qu'intervenant ce que Schwartz présente comme la deuxième 
partie des techniques transitives, à savoir celles qui concourent à la résolution des problèmes.

Le travail axé sur les projet a permis à chacune une authenticité dans l'expression de ses préoccupations en raison du climat de confiance présent dans le groupe. À l'analyse des écrits et des débats concourant à l'élaboration des projets, les travailleuses sociales ont fait part de leurs pratiques professionnelles respectives, ce qui a conduit au partage des façons de faire de chacune.

La participation de l'ensemble des professionnelles à l'élaboration des projets de chacune a rempli plusieurs fonctions :

- elle a contribué tout d'abord à l'apprentissage d'une méthode de travail, en multipliant les opportunités de construction de projets; c'est en faisant que l'on apprend;

- $\quad$ elle leur a permis de réaliser que, pour qu'un groupe se perpétue, il faut que chaque membre y trouve son intérêt; ainsi, une travailleuse sociale qui ne construisait pas de projet croyait sa présence dans le groupe remise en question;

- les professionnelles ont pris conscience de leur volonté commune de trouver une réponse aux problèmes des usagers qui soit en cohérence avec leur identité professionnelle.

\section{L'ÉVALUATION DU TRAVAIL}

L'évaluation du travail fait au cours de ces rencontres s'est déroulée en deux temps; le premier, avec les travailleuses sociales et l'intervenant, le second avec les encadrantes techniques.

\section{Premier temps de l'évaluation}

Dresser un premier bilan a pour objectif que les participants du groupe s'approprient l'évaluation de leur action et ne laissent pas ce soin à d'autres, qui y sont étrangers. En partant du contrat que nous avions passé ensemble, je leur ai proposé d'exprimer leurs impressions quant à l'application et au respect de ce contrat. Voici les réactions des cinq travailleuses sociales.

A. « Je trouve que le contrat est rempli; tout ce qui est notifié dans le contrat, on l'a fait. C'était adapté au travail de terrain et à notre demande sur le plan de la méthodologie. » 
B. “Cela nous a aidés à élaborer l'ébauche du projet, cela a aidé également dans l'écriture par les échéances qui étaient imposées. Le groupe, ça a aidé, questions et clarifications, la spécificité des populations de chacune m'a interrogée, cela m'a aidée et continue de le faire. Trois heures c'est frustrant, l'apport méthodologique était cependant indispensable. "

C. "Le fait de nous voir dans ce cadre nous a évité de nous retrouver dans le cadre de "l'action collective". Il est vrai que vu où j'en suis dans mon projet, je ne vois pas comment le mettre en place sans le travail que l'on fait entre nous. "

D. (qui a été absente pendant la moitié du temps pour des raisons de santé) “ Je pense que je vais stopper ce projet, ma longue absence m'a fait décrocher. Par ailleurs je vais quitter le secteur. Par conséquent il est vrai que mon investissement est moindre. "

E. " ll est très important de nous retrouver pour évoquer nos pratiques, le côté inter-institutionnel de notre groupe est une richesse et développe un espace de liberté. "

Pour la suite à donner, quatre participantes ont exprimé l'intention de poursuivre dès que possible. L'une d'elles a ajouté qu'en plus de notre travail elle souhaite bénéficier d'une formation au travail social de groupe. Une autre a exprimé le besoin d'aller plus loin sur le plan méthodologique. Ce besoin de formation fut réaffirmé par les deux autres participantes.

\section{Second temps de l'évaluation}

C'était la première fois que les encadrantes des différents services se rencontraient. Au départ le bilan établi au premier temps leur a été présenté, puis les professionnelles ont exposé chacune leur tour les projets qu'elles ont écrits. Les encadrantes se sont montrées fortement intéressées par les projets et elles ont convenu à l'unanimité du travail accompli et de l'intérêt de le poursuivre.

Les encadrantes ont mis en évidence la richesse d'un groupe pluri-institutionnel qui fait preuve d'une cohérence de l'intervention dans un secteur géographique. Elles ont également noté la maturité du groupe qui a pu fonctionner en respectant la spécificité de chaque institution. 
Les travailleuses sociales ont insisté sur leur souhait de bénéficier d'une formation propre au travail social de groupe.

La réunion s'est terminée par un engagement de la part des encadrantes de faire en sorte que les institutions se fassent le relais de la demande de formation des travailleuses sociales, en apportant leur appui.

\section{L'ANALYSE DU PROCESSUS DE CONSTITUTION ET DE RECONNAISSANCE DU GROUPE DE TRAVAILLEUSES SOCIALES}

\section{État des lieux et des personnes}

L'intervention qui a été décrite concerne un groupe de travailleuses sociales qui se rencontrent sur un territoire géographique commun au sujet d'une population d'usagers qui sont touchés par un dispositif social commun. Dans le cadre de ce dispositif, les acteurs sont multiples et proviennent d'horizons différents tant par leurs positions (payeurs, décideurs, exécutants) que par leurs fonctions professionnelles (rapporteurs, financiers, techniciens, responsable de circonscription, travailleurs sociaux, usagers).

Devant la lourdeur de leur tâche, les travailleuses sociales qui sont au contact des usagers éprouvent un besoin de se regrouper. D'où peut bien venir ce besoin de se retrouver? Et pour quoi faire?

Bien qu'elles soient d'institutions différentes, les travailleuses sociales ne peuvent s'éviter dans la mesure où elles sont convoquées aux réunions de la CLI (Commission locale d'insertion), où sont débattus les dossiers des bénéficiaires qu'elles présentent.

Au milieu des membres de la Commission qui doivent statuer sur les attributions des allocations, les travailleuses sociales sont les seules personnes qui connaissent les demandeurs. Elles ont donc une place particulière puisqu'elles parlent au nom des usagers.

Elles se situent en interface entre les bénéficiaires et la CLI. Les usagers leur accordent un pouvoir de décision qu'elles ne possèdent que partiellement. Par ailleurs, aux yeux des membres de la Commission, dans la mesure où la travailleuse sociale représente le demandeur elle est plus ou moins inconsciemment assimilée à celui-ci. 
Dans un cas comme dans l'autre, la travailleuse sociale peut éprouver des difficultés à se positionner. Le malaise qui résulte d'une telle situation engendre une insatisfaction. À la Commission ne sont débattus que des dossiers, alors que la travailleuse sociale évoque des personnes qu'elle rencontre et dont elle connaît la situation précaire.

Tous les secteurs d'activité et toutes les professions ont leur langage, leurs valeurs et bien d'autres particularités qui font que des individus qui se réclament d'une même spécificité professionnelle se reconnaissent, se comprennent, se regroupent. Les travailleurs sociaux n'y échappent pas; dans le cadre du dispositif d'insertion, les partenaires sont multiples et représentent par leurs fonctions des intérêts divergents. En référence à cette différence, la travailleuse sociale peut ressentir un relatif isolement.

II y a aussi un aspect du fonctionnement du dispositif qui peut mettre en difficulté les professionnelles du social; il s'agit de la demande de l'usager, du besoin que la travailleuse sociale perçoit chez cette personne et de la contractualisation souvent " bidon ", faute de temps, qui ponctue son travail. La demande faite aux travailleurs sociaux (instruction de dossiers et contractualisation) serait-elle en décalage avec leur culture, voire leur éthique professionnelle? C'est une hypothèse. Plus exactement, le manque de moyens dont dispose le travailleur social pour répondre aux situations de plus en plus dégradées des usagers ne le met-il pas en porte-à-faux? Son travail est-il bien en congruence avec son identité professionnelle?

Dans la situation qui nous occupe, nous avons vu que les travailleuses sociales bénéficiaient, à l'exception de celle du CCAS, d'un encadrement technique soutenu. Cependant, elles ont malgré tout éprouvé le besoin de se regrouper pour produire quelque chose qui améliore la qualité du service aux usagers. C'est du moins le sens qu'elles donnent principalement à leur regroupement.

Nous pensons, toutefois, y voir une démarche normale de regroupement si l'on se réfère aux écrits de Castoriadis (1974), qui précise à propos des regroupements de travailleurs que ce sont « des groupes élémentaires où se développent des sentiments fraternels, des éléments de solidarité ", qu'ils " sont des regroupements de production et de lutte ". 
Ce besoin de mettre en place une recherche commune afin d'améliorer la réponse aux usagers peut trouver sa justification dans ces quelques phrases de Castoriadis : « Le groupe informel n'est ni un lieu où se jouent uniquement des relations de sympathie, ni un lieu d'activité où sont en œuvre des tendances à la réalisation de soi déconnectées du travail quotidien [...] c'est au contraire l'endroit où s'expriment des sentiments de solidarité liée à la lutte. " Castoriadis évoque également, au sujet de la lutte que j'associe dans notre propos à la recherche de solutions et au besoin de faire connaître une réalité professionnelle pour le travailleur social et une réalité sociale pour l'usager, " qu'elle fait appel à l'inventivité, à la spontanéité et au dynamisme... "

Les travailleuses sociales se sont regroupées à la suite d'une réflexion sur le dispositif RMI. Elles avaient comme projet " de réfléchir à une autre dynamique de travail avec les bénéficiaires du RMI mais aussi entre les professionnels des services instructeurs ${ }^{1}$ ".

Elles se sont ainsi réunies pendant deux trimestres, faisant de l'information collective avant de passer à la deuxième phase de leur projet, à savoir « envisager un accompagnement social collectif des bénéficiaires dans l'élaboration de leur contrat, ainsi que des interventions ponctuelles sur des thèmes tels que le CES, la recherche d'emploi ".

Sans être vraiment clandestin, le groupe fonctionne à son initiative sans encadrement technique ou hiérarchique à qui rendre compte. Le groupe est une forme de travail envisagée par les travailleuses sociales pour appréhender différemment leur mission, pour améliorer la qualité du service rendu à l'usager.

C'est à ce moment qu'elles ont fait une demande extérieure à leurs institutions, sans en référer à ces dernières. Je suis donc arrivé dans ce groupe qui voulait mettre en place un travail social de groupe, sans savoir ce qu'était vraiment cet outil.

À la lumière des réflexions des travailleuses sociales, on peut faire certains constats. Le premier est que leurs préoccupations sont

1. Compte rendu de l'action collective d'information rédigée par les travailleuses sociales du pays de Retz.

2. Compte rendu de l'action collective d'information... Op. cit. 
essentiellement professionnelles. Ensuite, si l'encadrement dont elles bénéficient ne les satisfait pas, elles ont cependant l'accord pour se réunir et travailler collectivement. Leur rassemblement n'a pas pour objet de lutter contre leurs institutions, mais de se renforcer en savoirs, en savoir-faire professionnel, et également sur un plan narcissique. C'est probablement pour essayer de combler ce que l'encadrement existant n'apporte pas qu'elles recherchent hors cadre institutionnel une autre instance soutenante.

Notons à cet égard que, si l'encadrement ne répond pas aux besoins identifiés comme tels par les travailleuses sociales, il n'est pas pour autant inexistant ou volontairement inadéquat. Par ailleurs, il présente une certaine souplesse de fonctionnement, souplesse qui donne aux professionnelles la latitude pour travailler comme elles l'entendent.

\section{Le contrat}

Au départ, la demande du groupe à mon égard n'était pas claire; il a fallu un long travail de clarification avant que nous convenions du type de travail que nous allions faire. II a également fallu préciser ensemble le cadre dans lequel allait s'exécuter notre action. La préparation du contrat n'a pas été simple, puisque ce dernier n'a été retenu par tous que lors de notre troisième séance de travail. Cependant, le temps passé à affiner la demande collective n'a pas été, loin s'en faut, du temps perdu; il a permis à chacune de s'exprimer sur son malaise professionnel, sur la conception de sa mission, sur la réalité de ses prises en charge, sur les difficultés éprouvées dans la relation avec les usagers et sur les insatisfactions repérées dans les fonctionnements institutionnels.

Le contrat avec les professionnelles reprend donc la raison de ma présence au sein de leur groupe; il précise également les objectifs de notre travail et les modalités de celui-ci. Ce contrat est exclusivement axé sur la tâche. II reprend dans sa finalité sa demande initiale de travail social de groupe, puisqu'il prévoit la rédaction d'un projet d'intervention des travailleuses sociales. Il explicite également, et c'est l'un des éléments que j'ai appris du travail de clarification, la possibilité de faire s'exprimer les professionnelles sur leur pratique avec les bénéficiaires qui leur posent problème. Ce contrat laisse aussi apparaître la diversification des pratiques que les travailleuses sociales souhaitent mettre en place. 
Par ailleurs, afin de ne pas me mettre en concurrence avec leurs encadrantes techniques, je précise que la supervision proposée est de type pédagogique (donc axée sur l'acquisition d'une méthode spécifique), ce qui annonce que les domaines de compétence de chacun ne sont pas menacés.

Le contrat avec les professionnelles n'a pas été trop difficile à préparer, puisque je les ai rencontrées et que, partant des conclusions de notre travail, il m'a fallu rédiger un texte qui tienne compte de ces différents éléments. En revanche, le contrat à l'intention des institutions par l'intermédiaires des encadrantes respectives ne m'est pas apparu très simple à élaborer.

Tout d'abord, l'objet de ce contrat est de donner un cadre à mon intervention, c'est-à-dire que je ne viens pas travailler là dans une pièce avec des personnes qui sont de nulle part. Même si toutes ne sont pas dans les murs de leur institution, elles sont néanmoins présentes dans ce dispositif par l'institution ou le service qui les y envoie. II est donc indispensable d'informer ces institutions ou services que l'une de leurs salariées - voire plusieurs - participe à un regroupement de professionnelles.

II m'est apparu incontournable de rappeler à chaque institution que ce travail ne pouvait se faire que dans le respect des missions des institutions.

Il est difficile de passer contrat avec une personne ou un organisme que l'on connaît peu, ou que l'on connaît uniquement par ce que les professionnelles ont pu en dire, avec tous les états d'âme en présence, et les incertitudes que de tels énoncés supposent. Cependant, l'écrit que je leur ai soumis se présente comme une lettre informant qu'une de leurs salariées participait à une réflexion de groupe avec d'autres professionnelles d'autres institutions. Sans réaction particulière de leur hiérarchie, les travailleuses sociales pouvaient donc participer au travail de préparation et de réflexion quant à un projet de travail de groupe pour les usagers du RMI.

Par cette lettre d'information, j'ai en fait passer contrat avec les trois institutions dont sont issues les travailleuses sociales, sans qu'aucune institution ne m'ait fait de demande.

Quand, dans le cadre de la supervision, un contrat est passé, il est tripartite entre le superviseur, les supervisés d'une institution et 
l'institution elle-même. Ici, il s'agit d'une supervision de groupe pour laquelle un contrat avec chaque supervisé serait passé. Bien que cela puisse paraître paradoxal, la contractualisation ne peut se faire sans que chaque institution soit explicitement interlocuteur. Cependant, pour permettre une telle réalisation, on ne peut inclure dans le contrat la mission globale de l'institution, mais la spécificité d'une mission qui, elle, est commune aux trois institutions.

Au-delà d'une raison informative, qui dans un premier temps permet au groupe de travail de sortir de l'informel pour devenir existant, cette lettre lui donne une place officielle dans la série de mesures qui contribuent à remplir la mission des différentes institutions.

Dans la mesure où nous sommes dans une démarche quelque peu expérimentale, à un moment ou à un autre, il faudra bien évaluer le travail effectué. Cette évaluation devra se faire avec des représentants des différents services.

\section{La passation du contrat a été déclencheur de plusieurs facteurs}

Au-delà d'une contractualisation, qui a pour effet de lier différentes parties au vu d'objectifs à réaliser, la préparation du contrat a permis aux membres du groupe de travailleuses sociales de clarifier les attentes de chacune et d'exprimer les difficultés repérées par ce travail de clarification.

Il s'avère que ce n'est qu'à partir de ce moment que nous avons pu accéder à la phase de travail. Si le travail de réflexion, mené par chaque travailleuse sociale, a été aussi efficient, il est en partie à rattacher au travail préliminaire qui a défini de façon assez fidèle les centres d'intérêt de chacune.

Par ailleurs, les points du contrat étant clairs et en accord avec les attentes des travailleuses sociales, l'engagement de chaque protagoniste fait sens. La production des travailleuses sociales, tout à fait conforme au contrat, confirme la pertinence de ce dernier.

La participation des encadrantes techniques des trois institutions à l'évaluation finale montre aussi que le contrat était bien passé avec elles et que les résultats du travail réalisé leur importaient. Sans la passation d'un contrat avec les institutions, je doute fort 
qu'une évaluation commune, voire même fractionnée par institution, ait pu se tenir.

On peut constater que la participation des institutions à l'évaluation est la marque d'attentes de celles-ci. II apparaît aussi qu'elles ont l'idée qu'un travail s'est tenu dans le groupe et que ce travail correspond à leurs missions. II est donc évident que le contrat les a informées de l'objet de nos rencontres et que son contenu convenait aux différentes institutions, sans quoi il est vraisemblable que la participation des salariées aux rencontres aurait été menacée.

Lors de la rencontre évaluative, après la présentation générale du travail et des différents projets dans le détail, les encadrantes techniques ont réagi, par l'accord en commun qu'elles ont donné à une demande de formation commune aux membres de ce groupe, comme si le groupe des travailleuses sociales était en soi une entité. Qui plus est, ce groupe existe sans qu'elles l'aient provoqué, et elles se trouvent aujourd'hui à prendre des décisions pour que les demandes de ses membres aboutissent.

Ce qui est intéressant dans cette dynamique, c'est que chaque encadrante technique est censée s'exprimer au nom de sa ou de ses travailleuses sociales, alors qu'en fait c'est au sujet de la pérennité du groupe qu'elle le fait. C'est bien parce que chaque responsable voit un intérêt à ce que son salarié participe à ce groupe de travail qu'il en permet et en souhaite la persistance.

Un autre aspect que l'on peut retenir des différentes réactions des encadrantes techniques est en lien avec le fait qu'il est possible, voire satisfaisant dans un tel contexte pour des responsables d'être dessaisies (peu mais dessaisies quand même) d'une partie de leurs prérogatives sur leurs travailleuses sociales. De mon point de vue, il est deux cas de figure où il est possible d'accepter ce dessaisissement : 1) quand l'encadrante technique est peu préoccupée par l'objet en question, et 2) quand elle évalue, à un moment donné, que l'intervention la plus appropriée se passe en dehors de sa présence immédiate (le dessaisissement est alors tout relatif, car il est de la volonté de l'encadrante technique qui peut interrompre le processus à tout moment). Un tel fonctionnement demande une confiance mutuelle entre les travailleuses sociales et leurs hiérarchies, mais le cadre défini dans le contrat donne également les règles qui en permettent la mise en place. 
Dans ce système complexe, où chacun tente de se situer au mieux, les travailleuses sociales dans la réponse à apporter aux usagers, les encadrantes techniques dans leur fonction de management, avec en deuxième contrainte la mission de l'institution qu'elles représentent, et le superviseur dans son accompagnement des travailleuses sociales dans la rédaction de leur projet, on peut formuler quelques remarques dont certaines ne sont pas sans paradoxes.

Les encadrantes techniques assument pleinement leur tâche en déléguant l'encadrement du groupe à une personne qui n'est pas encadrante.

Les encadrantes techniques ont accepté un contrat pour un travail qu'elles n'ont pas sollicité, mais qui a été demandé par leurs travailleuses sociales, au nom de la réponse à apporter aux usagers.

La personne qui aide le groupe dans sa réflexion, qui l'encadre, ne possède pas seule le pouvoir quant à l'existence de ce groupe. Ce pouvoir appartient également à des membres extérieurs au groupe de travail: les encadrantes techniques (c'est peut-être en partie la raison pour laquelle les hiérarchies respectives ont pu accepter sa mise en place).

Il est possible que les encadrantes techniques aient laissé leurs travailleuses sociales participer à ce groupe de travail parce qu'aucune d'entre elles n'en avait la responsabilité. (II est à noter que lorsque l'évaluation d'un travail n'est pas probante, ou pire qu'elle est très discutable, peu de personnes se battent pour en revendiquer le commandement.) Cependant cette idée selon laquelle il y a une indépendance du groupe des professionnelles vis-à-vis des institutions est à retenir, car, en autorisant les travailleuses sociales à participer à ce groupe, les institutions partagent le fait de ne pas connaître, les unes plus que les autres, l'ensemble des solutions à apporter pour répondre aux besoins des usagers.

La non-appartenance à une institution (annoncée comme telle) du groupe évite aussi aux institutions de refuser la participation de leurs professionnelles à une instance qui pourrait complètement leur échapper (en étant sous la houlette d'une autre institution). Dans le cas qui nous occupe, le contrat, tel qu'il est rédigé, permet à chaque institution de se positionner comme décideur de la réalisation du projet, sans en référer aux autres institutions. 
La non-appartenance du groupe de travailleuses sociales est à relier avec les institutions dont elles dépendent, car en fait il y a bien une appartenance à quelque chose : à la mission qui justifie leur engagement commun, le dispositif du RMI. En axant principalement le contrat sur la tâche des professionnelles en lien avec la mission spécifique du RMI, les différents services pouvaient difficilement refuser à leurs salariées d'y prendre une place, d'autant que l'encadrement qu'ils proposent s'avère insuffisant ${ }^{3}$.

La durée du contrat, six mois (six séances de travail), ne représentait qu'un moment limité de part et d'autre et permettait ainsi un engagement tout à fait relatif des institutions. Cependant, lors de la phase d'évaluation, qui a somme toute été positive pour l'ensemble des professionnelles (bilan dressé par les encadrantes techniques) ${ }^{4}$, les institutions respectives se sont réapproprié le groupe pour qu'il accède à une formation commune. Les encadrantes techniques représentant les trois institutions se sont engagées, au nom de leur service, à faire ce qui était en leur pouvoir non seulement pour que les professionnelles puissent bénéficier de la formation qu'elles demandaient, mais pour que toutes les travailleuses sociales du groupe qui le désiraient aient accès à cette formation.

Avant cette rencontre évaluative, les encadrantes techniques ne se connaissaient pas et, après une heure et demie passée ensemble pour la première fois, elles ont convenu d'une action commune.

Durant toute la phase de construction du groupe qui a conduit à la phase finale, dont nous venons de présenter une partie des effets, rien ne s'est passé dans la facilité. Notamment en ce qui concerne les premières rencontres où le collectif de pairs a dû faire « le deuil » de son modèle d'identité, en acceptant que ses membres

3. II ne s'agit pas ici d'un jugement de valeur des encadrements techniques (j'en serais bien incapable), mais seulement d'un constat, que l'on pourrait peut-être élargir à d'autres institutions ou services qui ont à traiter de ce dossier, constat dont on ne peut se réjouir de toute façon.

4. Dire que le bilan a uniquement été dressé par les encadrantes techniques est insuffisant, car les travailleuses sociales ainsi que moi-même avons déclaré que nous faisions une évaluation positive de l'action menée et que nous estimions le contrat rempli. Cependant, si les institutions n'avaient pas fait le même constat que nous, la suite donnée à ce groupe eût été sûrement différente. 
pouvaient être différents, surtout dans leurs représentations professionnelles. À cette phase du travail, les tensions ont été nombreuses entre les travailleuses sociales; la chute du monolithe n'a pas été sans les fragiliser (même si la différenciation pouvait se faire parce que le groupe avec une personne extérieure permettait ce changement). Les relations des travailleuses sociales avec leurs encadrements techniques n'ont pas toujours été simples. De toutes ces tensions est née une nouvelle dynamique qui a permis aux institutions et à leurs salariées de créer une synergie, d'introduire des pratiques plus adaptées aux usagers.

Cependant, si ces tensions ont contribué au changement de regards portés sur le dispositif par les professionnelles et leurs institutions, il est d'autres tensions que l'on peut également associer à ce travail : ce sont celles du superviseur (bien que le terme ici ne me semble pas des plus appropriés). Tensions qui se sont tissées, perceptiblement ou non, vis-à-vis des travailleuses sociales, de leur encadrement et en son for intérieur.

\section{Le rôle du superviseur dans un tel processus}

En fait, il ne s'agit pas d'un rôle, mais d'une succession de rôles dont il faut, en fonction des phases, dialectiser la prédominance.

Le rôle de clarificateur, indispensable tout le temps, mais ô combien déterminant lors de la phase d'élaboration du contrat, a représenté un gros investissement de ma part. La préparation des questionnements, afin de les arrimer avec les préoccupations de chacune et avec la demande du groupe, m'a beaucoup intéressé. C'est peutêtre également parce que de ce travail préliminaire dépend la teneur du contrat et du travail qui sera réalisé avec les professionnelles. Cependant, durant la phase de travail comme à celle de l'évaluation, il est toujours nécessaire d'aider les individus à clarifier leurs objectifs, leurs intentions, leurs motivations.

Le rôle de facilitateur de la parole des professionnelles a aussi toute sa place dans l'énumération des différents rôles que le superviseur doit assumer pendant ce travail. Ce rôle de facilitateur se traduit par une attitude d'aide relative à l'expression par le professionnel de ses difficultés. Il est alors important de se centrer sur ce qui fait problème, et de l'entendre comme problème pour le professionnel. Faciliter la parole, c'est encourager la personne qui présente 
un cas, une difficulté, un problème à poursuivre son exposé jusqu'à son terme, c'est-à-dire lui permettre d'aller jusqu'à sa question.

L'animation du groupe ne se fait pas seul, ce rôle revient de fait au superviseur qui fait circuler la parole, interroge, partage le temps de parole, hiérarchise les points à aborder, recherche le feed-back...

La demande initiale comprenait de l'aide pour la mise en place d'un travail social de groupe. Sans une pratique de ma part dans ce domaine, il m'eût été impossible de travailler avec ce groupe comme cela a été le cas. J'ai dû, durant la phase de travail ainsi que durant la phase de clarification, présenter différents aspects du travail de groupe que l'on ne peut connaître sans une pratique de cette méthode.

J'ignore si la pédagogie utilisée a été adéquate. Ce que je sais, par contre, c'est que les quelques apports méthodologiques que j'ai pu faire dans ce domaine ont été retenus, et même mis en pratique, au moins dans la conception des projets.

Dans un tel groupe de travail, le superviseur doit évaluer la progression des professionnelles par rapport à l'objectif initial, en l'occurrence la rédaction d'un projet de travail de groupe pour les usagers. L'évaluation du superviseur intervient à plusieurs reprises au cours du fonctionnement du groupe : notamment à la phase de présentation des problématiques ainsi que lors de la rédaction de ces projets, pour ce qui est des retours faits à chaque travailleuse sociale. J'ai accordé beaucoup d'importance à ce que le travail effectué par les travailleuses sociales participe à la mission des services représentés dans le groupe.

Au-delà de l'aspect technique de superviseur dans ce système, mon apport a surtout été d'identifier les différents systèmes dans lesquels travaillent les professionnelles, d'en repérer les points communs à travers la parole des travailleuses sociales pour ensuite orienter leurs motivations, leur créativité vers une dynamique nouvelle. Exprimé ainsi, c'est sûrement un peu réducteur. Cependant, ce n'est qu'à partir des éléments apportés par les professionnelles que j'ai pu construire des analyses, faire des rapprochements, élaborer des hypothèses. Bien sûr, il faut aider à clarifier les intentions et les objectifs, mais dans l'exemple qui nous intéresse les travailleuses sociales insufflaient un tel désir de trouver de nouvelles pratiques, d'innover que mon rôle, assurément indispensable, doit cependant 
demeurer modeste. La volonté qu'un projet aboutisse de la part des encadrantes techniques révèle (malgré tous les conflits internes inhérents à toutes les institutions) également des conditions favorables à une réalisation.

Le processus était déjà en œuvre avant mon intervention dans le groupe; l'appel des travailleuses sociales à une personne extérieure à leurs institutions venait pointer une insatisfaction ou plutôt un manque. En fait, ces professionnelles sont allées chercher hors institution de quoi " s'institutionnaliser ", ou plutôt de quoi institutionnaliser une pratique. Car, à la fin de ma participation dans le groupe, les encadrantes techniques se sont réapproprié la conduite du groupe. $\mathrm{Si}$, par la suite, je suis amené à travailler à nouveau avec ce groupe, ce ne sera qu'à la demande des institutions en présence.

En synthèse, cette action montre que par un travail de groupe de professionnelles il y a moyen de faire au-delà de nos barrières institutionnelles un travail d'alliances qui profite aux usagers.

Christophe DALIBERT

Éducateur spécialisé Institut de formation sociale des Yvelines

Pontoise, France

\section{Références bibliographiques}

CASTORIADIS, J. (1974). “Socialisme et Barbarie », dans L'expérience du mouvement ouvrier, T. 2, 10/18, p. 43.

SHULMAN, L. (1976). Une technique de travail social avec des groupes. Le modèle de médiation, Paris, E.S.F. 